\title{
Infos zur Pflegekammer frei Haus
}

\section{DBfK Nordwest bietet Veranstaltungen an}

") Der Deutsche Berufsverband für Krankenpflege (DBfK) Nordwest bietet Informationsveranstaltungen rund um Pflegeberufekammern an. Pflegende oder Einrichtungen können sich die Experten kostenlos zu einem Vortrag ins Haus holen. Gut drei Stunden dauern diese Veranstaltungen, der Berufsverband bringt alle Materialien mit.

„Hinter dem Angebot steht die Überzeugung, dass nur informierte Pflegende sich eine objektive Meinung zur
Pflegeberufekammer bilden können. Diese Erfahrung hat der DBfK in anderen Bundesländern gemacht, in denen die Selbstverwaltung bereits auf den Weg gebracht wurde", so Martin Dichter, Vorsitzender des DBfK Nordwest. Es gehe aber auch darum, beruflich Pflegenden Lust auf Politik und Beteiligung zu machen und sie so zu ermutigen, sich in den Pflegeberufekammern zu engagieren. „Deshalb ist es uns wichtig, unkompliziert Veranstaltun- gen in allen fünf Bundesländern (Bremen, Hamburg, Niedersachsen, Nordrhein-Westfalen, Schleswig-Holstein) unseres Regionalverbandes anzubieten - unabhängig davon, wie der Stand der Entwicklung hinsichtlich einer Pflegeberufekammer in dem jeweiligen Bundesland ist", erklärt Swantje Seismann, Mitglied des Vorstandes des Berufsverbandes.

\section{www.dbfk.de}

\section{Mangelware Intensivpflegekräfte}

\section{Deutsche Krankenhausgesellschaft legt Gutachten vor}

» Den Kliniken gehen die Intensivpflegekräfte aus. Jedes zweite Krankenhaus (53\%) hat Probleme, entsprechende Stellen zu besetzen. Bei den großen Häusern sind es sogar 68\%. Das geht aus einem Gutachten hervor, das die Deutsche Krankenhausgesellschaft (DKG) in Berlin präsentiert hat. Insgesamt sind bundesweit 3.150 Stellen für Intensivpflegekräfte offen. Die vorgegebene Fachkraftquote von min- destens 30\% erreichen drei Viertel der Intensivstationen.

Aus Sicht der DKG ist die Patientenversorgung dennoch „objektiv gut“. Eine Pflegevollkraft versorgt laut Gutachten pro Schicht durchschnittlich 2,2 Patienten. Die von der Deutschen Interdisziplinären Vereinigung für Intensiv- und Notfallmedizin (DIVI) geforderte Quote von zwei Fällen pro Schicht werde damit im Mittel er-

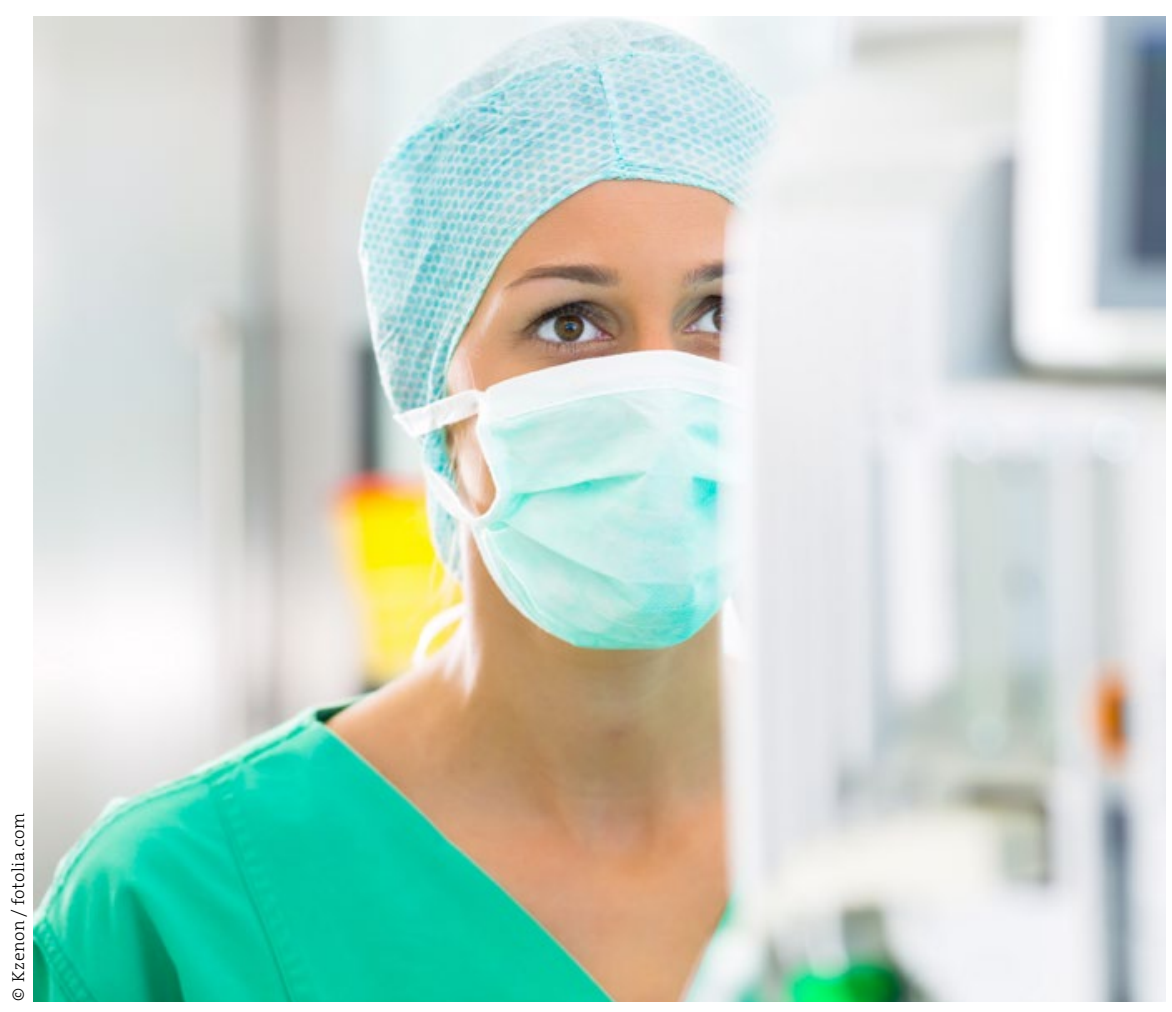

reicht. Die DKG sieht vor allem Politik und Kostenträger in der Pflicht, dem Fachkräftemangel zu begegnen. „Wer mehr Personal und Personaluntergrenzen fordert, muss auch die Refinanzierung sichern", so DKG-Präsident Thomas Reumann.

Zweifel an der guten Versorgungssituation hegen sowohl Gewerkschaften als auch Pflegeverbände. „Nichts ist gut in der Intensivpflege“, kritisiert Sylvia Bühler, Bundesvorstandsmitglied von Verdi. Aus Berichten von Beschäftigten und vorliegenden Gefährdungsanzeigen werde deutlich, dass ein Verhältnis von 1:3 Pflegekraft-zuPatienten eher die Regel als eine Ausnahme sei. „Statt den Fachkräftemangel in der Pflege zu beklagen, muss zügig gehandelt werden", erklärt Bühler mit Verweis auf die Arbeits- und Ausbildungsbedingungen. Die DKG sei bei den Verhandlungen zu Personaluntergrenzen gefordert, endlich Nägel mit Köpfen zu machen. Notwendig sind aus Sicht der Gewerkschaft Vorgaben, die Sicherheit für die Patienten und Entlastung für die Beschäftigten bringen.

Die Grundlage für das Gutachten der DKG bildeten die Daten von bundesweit 314 Krankenhäusern. Diese gaben im Herbst 2016 Auskunft zur Personalsituation auf ihren Intensivstationen. 